\title{
Ischemia of the lateral femoral cutaneous nerve during periacetabular osteotomy using Smith-Petersen approach
}

\author{
Takahiko Kiyama • Masatoshi Naito • \\ Kei Shiramizu · Tuyoshi Shinoda · Akira Maeyama
}

Received: 9 January 2009/Accepted: 7 June 2009/Published online: 24 June 2009

(C) Springer-Verlag 2009

\begin{abstract}
Background Lateral femoral cutaneous nerve (LFCN) injury is a common complication in the Smith-Petersen approach to the hip. This complication may be induced by neural ischemia or direct trauma during the procedure. The purpose of this study was to investigate the relationship between the neural ischemia of LFCN and postoperative sensory disturbance.

Materials and methods Nineteen patients who underwent periacetabular osteotomy through the Smith-Petersen approach were investigated. To evaluate neural ischemia, we measured the blood flow of LFCN using a laser Doppler flowmetry. The measurements were performed before and after osteotomy at the point $1 \mathrm{~cm}$ distal from the lower border of the inguinal ligament. LFCN was retracted to the medial side during the procedure. There was no direct trauma to LFCN in all cases. Postoperative sensory disturbance was evaluated at 2 weeks, 3 months, and 1 year follow-up after surgery.

Results After osteotomy, the blood flow of LFCN was decreased to 2.4 from $3.3 \mathrm{ml} \mathrm{min}{ }^{-1} 100 \mathrm{~g}^{-1}$ when compared with that before osteotomy $(P<0.01)$. Postoperatively, 14 of 19 patients had sensory disturbance at 2 weeks, 8 of 19 patients at 3 months, and 2 of 19 patients at 1 year follow-up. The blood flows of both patients who had persistent symptoms over 1 year after surgery had been decreased by more than $50 \%$ during operation.
\end{abstract}

T. Kiyama $(\bowtie) \cdot$ M. Naito $\cdot$ K. Shiramizu $\cdot$ T. Shinoda .

A. Maeyama

Department of Orthopaedic Surgery,

Fukuoka University School of Medicine,

7-45-1, Nanakuma, Jonan-ku, Fukuoka 814-0180, Japan

e-mail: wave.kiyataka@excite.co.jp; wavekiyataka@yahoo.co.jp
Conclusions Decrease of blood flow of LFCN by more than $50 \%$ seems to cause persistent symptoms after surgery through the Smith-Petersen approach even if direct trauma to the nerve is avoided. Excessive traction by retractors is thought to be the main cause of blood flow reduction.

Keywords Nerve $\cdot$ Blood flow $\cdot$ Traction

\section{Introduction}

In 1917, the Smith-Petersen approach was designed for access to the hip joint and involves external iliac dissection [11]. In 1949, he extended this approach to give access to the anterior hip capsule and lateral femoral anatomy [12]. Since then, this approach has been widely used as an excellent approach to the hip.

Lateral femoral cutaneous nerve (LFCN) injury is a relatively frequent complication in the Smith-Petersen approach to the hip. Injury to the LFCN can occur during surgery, resulting in an altered sensation known as meralgia paresthesia. This complication seems to be induced by direct injury or neural ischemia during operation to the hip. Regarding the relationship between neural ischemia and nerve function, Lundborg [6] reported that complete neural ischemia is followed a deterioration of nerve function. Also, we experienced that some patients had sensory disturbance after periacetabular osteotomy through this approach even if direct injury to the nerve was avoided. Therefore, in order to investigate the relationship between the neural ischemia of LFCN and postoperative sensory disturbance, we measured the nerve blood flow during procedures using the Smith-Petersen approach. 


\section{Materials and methods}

A total of nineteen patients who underwent curved periacetabular osteotomy [8] through the Smith-Petersen approach were investigated. This study was approved by our institutional ethics committee and conformed to the Declaration of Helsinki. All patients signed the approved informed consent. Patients who had damage to the LFCN or its branches were not included in this study. In a curved periacetabular osteotomy, the Smith-Petersen approach is used for surgical exposure [8]. An anterior incision approximately $12 \mathrm{~cm}$ long begins over the iliac crest $5 \mathrm{~cm}$ posterior to the anterior superior iliac spine (ASIS), extends forward along the iliac crest to the ASIS, and then turns distally for $7 \mathrm{~cm}$ along the groove between the tensor fasciae late and the sartorius muscle. The LFCN is dissected free from the surrounding connective tissue and is retracted to the medial side with sartorius muscle during the procedure. The data collected for analysis were age, gender, body-mass index (BMI), and surgical time. BMI was defined as weight in kilograms divided by height in meters squared $\left(\mathrm{kg} / \mathrm{m}^{2}\right)$. There were 3 men and 16 women. Average age at the time of operation was $35.9 \pm 10.2$ years (range, $14-55$ years). Average BMI was $23.5 \pm 18.1 \%$ and average surgical time was $73.7 \pm 10.7 \mathrm{~min}$.

Intraoperative measurements of nerve blood flow

A laser Doppler flowmeter (ALF 21N; ADVANCE, Tokyo, Japan) was utilized to measure the intraoperative blood flow of the nerve (Fig. 1) [3, 4]. To avoid inaccurate measurements caused by bleeding from the surrounding tissue, a plastic tube was placed on the tip of the probe [4]. The measurements were performed before and after osteotomy at the point $1 \mathrm{~cm}$ distal from the lower border of the inguinal ligament. The blood flow measurements were repeated three times, and mean values were calculated. Values measured by laser Doppler flowmetry refer to the relative change in red blood cell movement. A decrease ratio for the nerve blood flow was calculated for each case according to the following formula: decrease ratio $=(a-b) / a \times 100 \quad(\%)$, where $a=$ blood flow before osteotomy and $b=$ blood flow after osteotomy. In all cases, the same investigator (T.K.) took the blood-flow measurements.

\section{Clinical evaluation}

Postoperative sensory disturbance in the LFCN distribution was evaluated at 2 weeks, 3 months, and 1 year follow-up after surgery. Von Frey pressure testing was performed on all affected hips using the Semmes-Weinstein

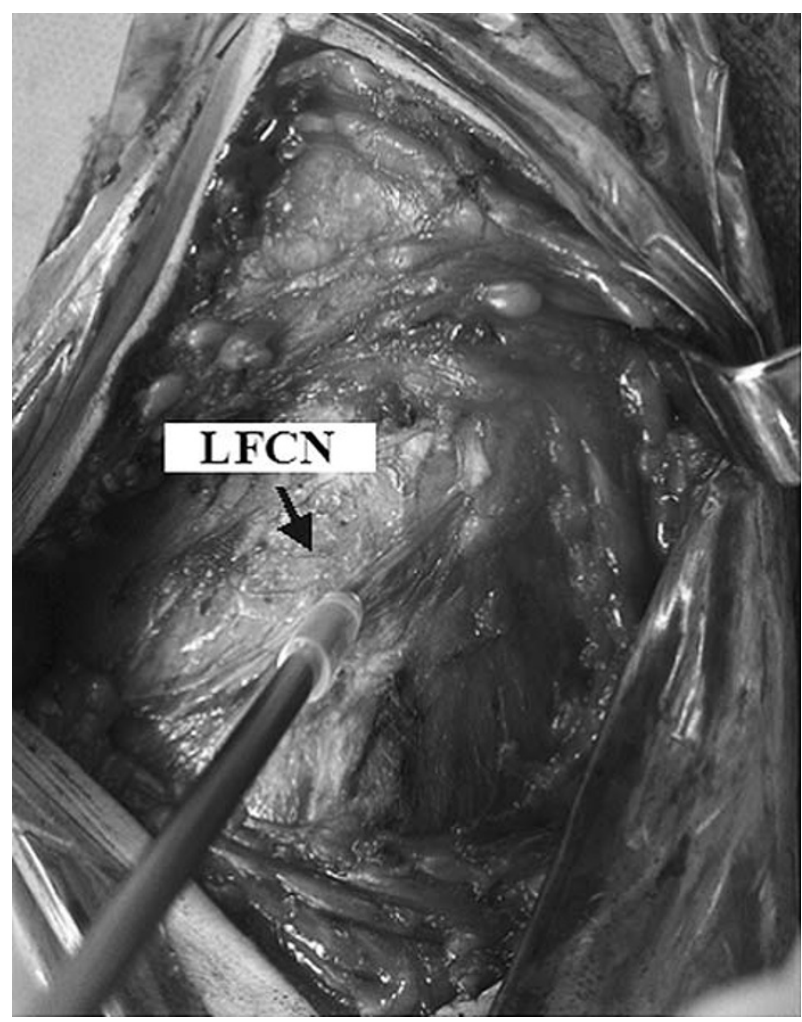

Fig. 1 Intraoperative measurements of nerve blood flow. The measurements were performed before and after osteotomy at the point $1 \mathrm{~cm}$ distal from the lower border of the inguinal ligament

monofilament. Normal values were considered 3.22 or below [2]. The evaluation was performed by one of our authors (A.M.) who was blinded to the results of bloodflow measurements.

The Wilcoxon signed rank test and the Pearson correlation coefficient were used for statistical analyses with the significance level at $P=0.05$. A simple linear regression analysis showed a $95 \%$ confidence level with $n=19$ patients for detecting correlation among age, body-mass index, surgical time, and the decrease ratio.

\section{Results}

Intraoperative measurements of nerve blood flow

After osteotomy, the blood flow of LFCN was decreased to $2.4 \pm 0.9$ from $3.3 \pm 0.87 \mathrm{ml} \mathrm{min}^{-1} 100 \mathrm{~g}^{-1}$ when compared with that before osteotomy (Fig. 2$)(P<0.01)$. The mean decrease ratio was $23.5 \pm 18.1 \%$. The Pearson correlation coefficient analysis did not reveal significant associations of decrease ratio with age, body-mass index, and surgical time (Table 1). 


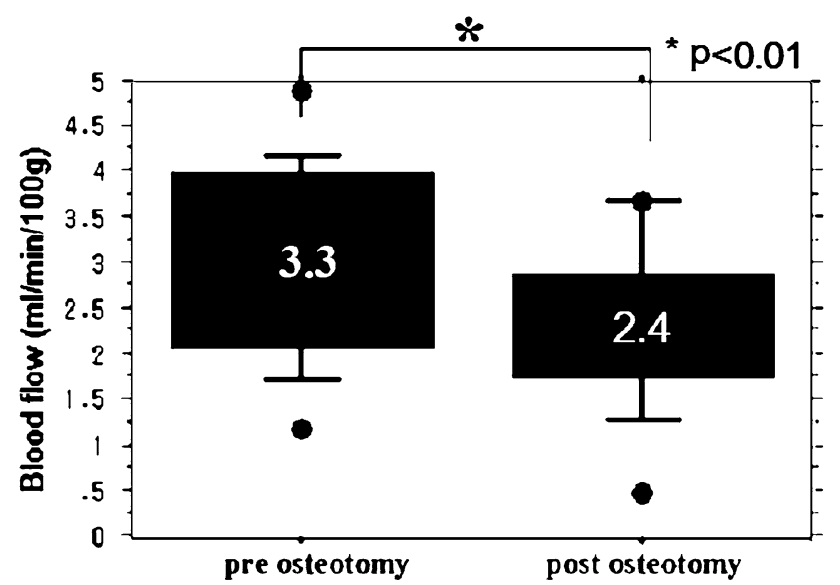

Fig. 2 Comparison of nerve blood flow before and after osteotomy. The nerve blood flow was decreased to $2.4 \pm 0.9$ from $3.3 \pm 0.87 \mathrm{ml}$ $\min ^{-1} 100 \mathrm{~g}^{-1}$ when compared with that before osteotomy $(P<0.01)$

Table 1 Results of regression analysis

\begin{tabular}{lll}
\hline & SRC & $P$ value \\
\hline Age & 0.02 & 0.95 \\
Body-mass index & 0.28 & 0.27 \\
Surgical time & 0.13 & 0.61 \\
\hline
\end{tabular}

SRC Standardized regression coefficients

\section{Clinical evaluation}

Postoperatively, 14 of 19 (74\%) patients had sensory disturbance by Semmes-Weinstein monofilament testing at 2 weeks, 8 of 19 (42\%) patients at 3 months, and 2 of 19 (11\%) patients at 1 year follow-up. The decrease ratios between patients with and without symptoms over 3 months after surgery were $35.2 \pm 16.6$ and $19.8 \pm 13.1 \%$, respectively $(P<0.01)$ (Fig. 3$)$. The blood flows of both patients who had persistent symptoms over 1 year after surgery had been decreased by more than $50 \%$ during the operation.

\section{Discussion}

A disadvantage related to the anterior approach to the hip for periacetabular osteotomy is a high incidence of lateral femoral cutaneous nerve dysesthesias. Hussell et al. [5] reported that the incidence of this complication morbidity with modified Smith-Petersen approach was approximately $30 \%$ of patients.

It has been reported that neural ischemia leads to perineural scar formation which will obstruct the progression of axonal sprouts, and procedures interfering with intraneural blood flow can be expected to induce disturbances

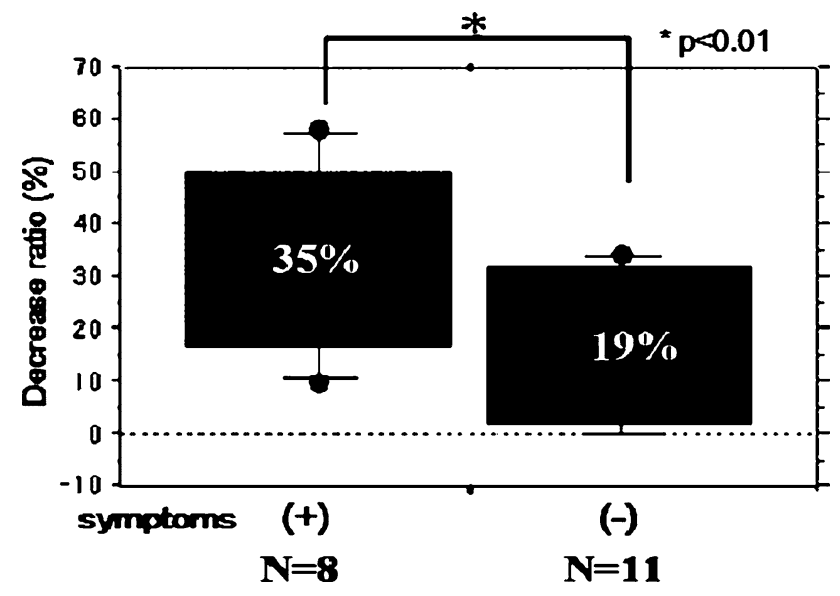

Fig. 3 Comparison of decrease ratio between patients with and without symptoms over 3 months. The decrease ratio of patients with symptoms over 3 months after surgery was significantly higher than that of patients without symptoms $(P<0.01)$

of nerve function [13]. Neural ischemia during surgery is thought to be induced by traction and compression to the nerve. When tension is applied to a nerve, both the extrinsic and the intrinsic vascular supplies are affected. In a basic study, Ogata et al. [9] demonstrated that the average stretching of more than $15.7 \%$ caused complete arrest of blood flow in the stretching nerve. Also, they reported that complete standstill of intraneural circulation was observed under compression of $50-70 \mathrm{~mm} \mathrm{Hg}$ or $60-80 \%$ of mean arterial pressure. Excessive traction or direct compression to the nerve can lead to nerve blood flow reduction and induce disturbances of nerve function.

In our clinical study, nerve blood flow was decreased by a mean of $27 \%$ during surgery. The decrease ratio of patients with persistent symptoms over 3 months after surgery was higher than that of patients without symptoms or with symptoms that disappeared in the early period. The blood flows of both patients who had persistent symptoms over 1 year after surgery had been decreased by more than $50 \%$ during the operation.

Many anatomical variations of the LFCN have been well known [1, 10]. Matthew reported that the distance along the inguinal ligament from anterior superior iliac spine to where the nerve crossed was approximately $36 \mathrm{~mm}$ (range, 6-73 mm) [7]. In the Smith-Petersen approach, the LFCN is retracted to the medial side with the sartorius muscle during the procedure. If this nerve runs more laterally, the forces applied to the nerve by retractors are higher. In the current study, a correlation did not reveal significant associations of decrease ratio with body-mass index and surgical time. Anatomical variations of the LFCN may be one of the factors that cause the reduction of nerve blood flow during surgery.

We note several limitations to our study. First, it involved only a small number of hips (19). Second, the 
LFCN is a relatively thin nerve. Therefore, this may hinder reliable and reproducible measurements of nerve blood flow. In this study, patients whose nerve was thinner than probe size at a measurement region were excluded. Finally, this study could not evaluate the relationship between anatomical variations of the LFCN and nerve blood flow. In future studies, an evaluation of the relationship between anatomical variations and change in blood flow is required.

In conclusion, the blood flow of the lateral femoral cutaneous nerve in the Smith-Petersen approach was significantly decreased during operation. The reduction of this nerve circulation can cause persistent symptoms after surgery using the Smith-Petersen approach even if direct trauma to the nerve is avoided. Excessive traction or direct compression to the nerve by retractors is thought to be the main factor of blood flow reduction.

Conflict of interest statement The authors declare that they have no conflict of interest related to the publication of this manuscript.

\section{References}

1. Bjurlin MA, Davis KE, Allin EF, Ibrahim DT (2007) Anatomic variation in the lateral femoral cutaneous nerve with respect to pediatric hip surgery. Am J Orthop 36:143-146
2. Dellon AL (1991) Operative nerve repair and reconstruction. JB Lippincott, Philadelphia, pp 135-158

3. Glenn JS, Richard JR, Julian HL (1986) Evaluation of laserDoppler flowmetry as a measure of tissue blood flow. J Appl Physiol 61:666-672

4. Hida S, Naito M, Kubo M (2003) Intraoperative measurements of nerve root blood flow during discectomy for lumbar disc herniation. Supine 1:85-90

5. Hussell JG, Mast JW, Mayo KA, Howie DW, Ganz R (1999) Comparison of different surgical approaches for the periacetabular osteotomy. Clin Orthop 363:64-72

6. Lundborg G (1973) Structure and function of the intraneural microvessels as related to trauma, edema formation and nerve function. J Bone Joint Surg Am 57:938-948

7. Matthew CG, Marston H, Anis O (2005) Lateral femoral cutaneous nerve. Clin Orthop 437:164-168

8. Naito M, Shiramizu K, Akiyoshi Y, Ezoe M, Nakamura Y (2005) Curved periacetabular osteotomy for treatment of dysplastic hip. Clin Orthop Relat Res 433:129-135

9. Ogata K, Naito M (1986) Blood flow of peripheral nerve effects of dissection, stretching and compression. J Hand Surg 11:10-14

10. Aszmann OC, Dellon ES, Dellon AL (1997) Anatomical course of the lateral femoral cutaneous nerve and its susceptibility to compression and injury. Plast Reconstr Surg 100:600-604

11. Smith-Petersen MN (1917) A new supra-articular subperiosteal approach to the hip. Am J Orthop Surg 15:592

12. Smith-Petersen MN (1949) Approach to and exposure of the hip joint for arthroplasty. J Bone Joint Surg Am 31:40-46

13. Starkweather RJ, Neviaser RJ (1978) The effect of devascularization on the regeneration of lacerated peripheral nerves. J Hand Surg 3:163-167 